\title{
Estimating the Population Standard Deviation with Confidence Interval: A Simulation Study under Skewed and Symmetric Conditions
}

\author{
Shipra Banik ${ }^{1}$, Ahmed N. Albatineh², Moustafa Omar Ahmed Abu-Shawiesh ${ }^{3}$ and \\ B.M. Golam Kibria ${ }^{4, *}$ \\ ${ }^{1}$ Department of Physical Sciences, School of Engineering and Computer Science, Independent University, \\ Dhaka 1229, Bangladesh \\ ${ }^{2}$ Department of Biostatistics, Florida International University, Miami, FL 33199, USA \\ ${ }^{3}$ Department of Mathematics, Faculty of Science, Hashemite University, Zarqa 13115, Jordan \\ ${ }^{4}$ Department of Mathematics and Statistics, Florida International University, Miami, FL 33199, USA
}

\begin{abstract}
This paper investigates the performance of ten methods for constructing a confidence interval estimator for the population standard deviation by a simulation study. Since a theoretical comparison among the interval estimators is not possible, a simulation study has been conducted to compare the performance of the selected interval estimators. Data were randomly generated from several distributions with a range of sample sizes. Various evaluation criterions are considered for performance comparison. Two health related data have been analyzed to illustrate the application of the proposed confidence intervals. Based on simulation results, some intervals with the best performance have been recommended for practitioners.
\end{abstract}

Keywords: Bootstrapping, Coverage probability, Interval estimator, Kurtosis, Robustness, Scale estimator, Skewed Distribution.

\section{INTRODUCTION}

A term often is used in the Inferential Statistics is known as confidence interval $(\mathrm{Cl})$. It measures the chance that a value will fall between a lower and an upper bound of a probability distribution. Given a $95 \%$ $\mathrm{Cl}$, say for an example, a stock XYZ's return will fall between $5.3 \%$ and $10.5 \%$ over the next year. It means that we are $95 \%$ confident that the return of holding $X Y Z$ stock over the next year will fall between $5.3 \%$ and $10.5 \%$. It is well-known that the most commonly used scale estimator, namely sample standard deviation (S) is very important in many statistical applications which provides a logical point estimate of the population standard deviation $(\sigma)$. The classical chisquare $100(1-\alpha) \% \mathrm{Cl}$ for $\sigma$ is based on the assumption that the underlying distribution of data is normal with no outliers. Unfortunately, this assumption for constructing $\mathrm{Cl}$ for $\sigma$ is very sensitive to normality assumption [1,2]. Many research papers in literature shows that $S$ is not an efficient scale estimator in skewed and/or leptokurtic distributions. As a result, it is not robust to slight deviations from normality, see [3] for details. Thus, when the parent population is far from normal, achieved confidence level of $\mathrm{Cl}$ of $\sigma$ is far from the

*Address correspondence to this author at the Department of Mathematics and Statistics, Florida International University, Miami, FL 33199, USA;

Tel: (305) 348 1419; Fax: (305) 348 6895; E-mail: kibriag@fiu.edu nominal level. Despite $S$ being the most efficient scale estimator for normal distribution and often used to construct the $100(1-\alpha) \% \mathrm{Cl}$ for $\sigma$ but the basic question is what happens if the data are not from a normal distribution but instead from heavier tails or from skewed distributions. Thus, the need for alternatives to the classical chi-square $100(1-\alpha) \% \mathrm{Cl}$ for $\sigma$ comes to play. Although much work has been done on improving $\mathrm{Cl}$ for population mean [4-7], improving robustness for $\mathrm{Cl}$ for $\sigma$ is still greatly unexplored.

The aim of this paper is (i) to evaluate and compare several available methods for constructing $\mathrm{Cl}$ for $\sigma$ and (ii) based on extensive simulation and numerical examples to seek evidences to recommend some $\mathrm{Cl}$ with best performance for researchers. Performances of the proposed methods are investigated through a Monte Carlo simulation study based on some evaluation criteria such as coverage probability, average width and SD of width. The coverage probability naturally varies from distribution to distribution for a given procedure, but a good procedure should keep this variation small. Furthermore, we want a $\mathrm{Cl}$ whose endpoints are generally close together, thus a small average width is good [8]. Two health related data are used to illustrate the results given in the paper. 
The organization of the paper is as follows. In section 2, different alternative methods for constructing $\mathrm{Cl}$ for $\sigma$ are presented. A Monte Carlo simulation study with flowchart is outlined in section 3. To illustrate findings of the paper, some real life data are analyzed in section 4. Finally, some concluding remarks and future research are presented in section 5 .

\section{METHODS FOR CONSTRUCTING CI FOR $\sigma$}

Suppose that $x_{1}, x_{2}, \ldots, x_{n}$ is a random sample of size $\mathrm{n}$ from the normal distribution, i.e. $X_{i} \sim N\left(\mu, \sigma^{2}\right)$ for all $i, \quad$ then $\quad \frac{(n-1) S^{2}}{\sigma^{2}}=\frac{1}{\sigma^{2}} \sum_{i=1}^{n}\left(X_{i}-\bar{X}\right)^{2} \sim \chi_{n-1}^{2}, \quad$ where $S^{2}=\frac{1}{n-1} \sum_{i=1}^{n}\left(X_{i}-\bar{X}\right)^{2}$ is the sample variance.

\subsection{Exact $\mathrm{Cl}$}

The classical chi-square $100(1-\alpha) \% \mathrm{Cl}$ for $\sigma$ is given as follows:

$$
L C L=\sqrt{\frac{(n-1) S^{2}}{\chi_{\frac{\alpha}{2},(n-1)}^{2}}} \text { and } \quad U C L=\sqrt{\frac{(n-1) S^{2}}{\chi_{1-\frac{\alpha}{2},(n-1)}^{2}}}
$$

Where $\chi_{\frac{\alpha}{2}}^{2}$ and $\chi_{1-\frac{\alpha}{2}}^{2}$ are the $(\alpha / 2)^{\text {th }}$ and $(1-(\alpha / 2))^{\text {th }}$ percentile points of the $\chi^{2}$ distribution with $(n-1) d f$.

\subsection{Robust CI}

The exact $\mathrm{Cl}$ for $\sigma$ in (1) is sensitive to minor violations of the normality assumption. Bonett [9] show that (1) has an asymptotic coverage probability of about $76,63,60$ and 51 for the Logistic, the Student $t(7)$, the Laplace and the Student $t(5)$ distributions respectively. These results are not very acceptable as symmetric distributions are not easily distinguishable from a normal distribution unless $\mathrm{n}$ is large. Also, the exact $\mathrm{Cl}$ for $\sigma$ in (1) as demonstrated by Lehman [10] is highly sensitive to the presence of outliers and/or to departure from normality.

Following above, [2] proposed an alternative to the exact $100(1-\alpha) \% \mathrm{Cl}$ for $\sigma$ based on [11] estimator, $\mathrm{Q}_{\mathrm{n}}$ [2]. A brief description of this method is given below:

Instead of assuming $X_{i} \sim N\left(\mu, \sigma^{2}\right)$, let $\mathrm{x}_{1}, \mathrm{x}_{2}, \ldots, \mathrm{x}_{\mathrm{n}}$ be a random sample of size $\mathrm{n}$ from a continuous, independent and identically distributed random variable. The $100(1-\alpha) \%$ robust $\mathrm{Cl}$ for $\sigma$ is as follows:

$$
L C L=\frac{D Q_{n}}{Z_{\frac{\alpha}{2}}+D_{1}} \text { and } \quad U C L=\frac{D Q_{n}}{Z_{1-\frac{\alpha}{2}}+D_{1}}
$$

where $D=1.28 \sqrt{n} * d_{n}$, An approximation result of $\mathrm{D}$ for larger values of $\mathrm{n}$ can be calculated as follows:

$D= \begin{cases}(1.28 \sqrt{n})\left(\frac{n}{n+1.4}\right) & \text { for odd values of } n \\ (1.28 \sqrt{n})\left(\frac{n}{n+3.8}\right) & \text { for even values of } n\end{cases}$

$D_{1}=1.28 \sqrt{n}, \mathrm{Q}_{\mathrm{n}}$ is the [11] estimator defined as $Q n=2.2219\left\{\left|X_{i}-X_{j}\right| ; i<j ; i=1,2,3, \ldots, n ; j=1,2,3, \ldots, n\right\}_{\{g\}}$, and $\left.g=\left(\begin{array}{l}h \\ 2\end{array}\right) \approx\left(\begin{array}{l}n \\ 2\end{array}\right) / 4\right) \quad$ where $\quad h=\left(\left[\frac{n}{2}\right]+1\right)$ (i.e., roughly half the number of observations). Here the symbol (.) represents combination and symbol [.] is used to take only integer part of a fraction. $Q_{n}$ estimator is the $g$-th order statistic of $\left(\begin{array}{l}n \\ 2\end{array}\right)$ inter-point distances. The value 2.2219 is chosen to make $Q_{n}$ a consistent estimator of scale for normal data (For values of $d_{n}$ see [2].

\subsection{Bonett $\mathrm{Cl}$}

Let $X_{i} \sim N\left(\mu, \sigma^{2}\right)$ for all $i$. [9] proposed the following $(1-\alpha) 100 \% \mathrm{Cl}$ for $\sigma$ :

$$
\mathrm{LCL}=\operatorname{sqrt}\left(\exp \left\{\ln \left(c \hat{\sigma}^{2}\right)-Z_{\alpha / 2} s e\right\}\right) \text { and } \mathrm{UCL}=
$$

$\operatorname{sqrt}\left(\exp \left\{\ln \left(c \hat{\sigma}^{2}\right)+Z_{\alpha / 2} s e\right\}\right)$,

where $Z_{\alpha / 2}$ is two-sided critical $z$-value, $s e=c\left[\left\{\hat{\gamma}_{4}(n-3) / n\right\} /(n-1)\right]^{1 / 2}, \quad \mathrm{c}=\mathrm{n} /\left(\mathrm{n}-\mathrm{Z}_{\alpha / 2}\right)$ and $\hat{\gamma}_{4}=n \sum_{i=1}^{n}\left(Y_{i}-\hat{\mu}\right)^{4} /\left(\sum\left(Y_{i}-\hat{\mu}\right)^{2}\right)^{2}$.

\subsection{Steve Large Sample Normal Approximations CI}

Steve [12] proposed the following $100(1-\alpha) \% \mathrm{Cl}$ for $\sigma$ :

$$
L C L=\sqrt{\frac{S^{2}}{1-Z_{\frac{\alpha}{2} \sqrt{\frac{\hat{\gamma}-1}{n}}}}} \text { and } \quad U C L=\sqrt{\frac{S^{2}}{1+Z_{\frac{\alpha}{2} \sqrt{\frac{\hat{\gamma}-1}{n}}}}}
$$

where $\hat{\gamma}$ is the kurtosis estimator. 


\subsection{Hummel, Banga and Hettmansperger Cls}

\subsubsection{Log asymptotic Approximation (Ln Asympt) $\mathrm{Cl}$}

Because of high skewness of the distribution $S^{2}$ for small $n$, [1] hereafter $\mathrm{HBH}$, using the method of applying natural $\log$ to $S^{2}$ in (4) (i.e. by adjusting skewness) proposed the following $100(1-\alpha) \% \mathrm{Cl}$ for $\sigma$ :

$$
\begin{aligned}
& L C L=\operatorname{sqrt}\left(s^{2} \exp \left(Z_{\frac{\alpha}{2} \sqrt{\frac{\hat{\gamma}-1}{n}}}\right)\right) \text { and } \\
& U C L=\operatorname{sqrt}\left(s^{2} \exp \left(-Z_{\frac{\alpha}{2} \sqrt{\frac{\hat{\gamma}-1}{n}}}\right)\right.
\end{aligned}
$$

\subsubsection{Adjusted Degrees of Freedom (AdjDF) Cl}

To find a better $\mathrm{Cl}, \mathrm{HBH}$ adjusted DF (i.e. (n-1)) of the exact $\mathrm{Cl}$ for $\sigma$ in (1) and proposed the following $100(1-\alpha) \% \mathrm{Cl}$ for $\sigma$ :

$L C L=\sqrt{\frac{\hat{r} S^{2}}{\chi_{\frac{\alpha}{2}, \hat{r}}^{2}}}$ and $\quad U C L=\sqrt{\frac{\hat{r} S^{2}}{\chi_{1-\frac{\alpha}{2}, \hat{r}}^{2}}}$

where $\hat{r}=\frac{2 n}{\hat{\gamma} e+\left(\frac{2 n}{n-1}\right)}$ and $\hat{\gamma} e$ is the estimate of kurtosis excess (e.g. for normal distribution $\hat{\gamma} e=\hat{\gamma}-3=0$ because $\hat{\gamma}=3$ for normal distribution) which is defined as

$$
\hat{\gamma} e=\frac{n(n+1)}{(n-1)(n-2)(n-3)} \sum_{i=1}^{n} \frac{\left(x_{i}-\bar{x}\right)^{4}}{S^{4}}-\frac{3(n-1)^{2}}{(n-2)(n-3)}
$$

If a random sample comes from the normal population, then $r=n-1$ and (6) reduces to (1).

\subsubsection{Modified Adjusted Degrees of Freedom (B-C AdjDF) Cl}

Due to low coverage probabilities of UCL of the AdjDF method (see equation 6), $\mathrm{HBH}$ adjusted only $100(1-\alpha) \%$ UCL in the following way:

$$
U C L_{B-C}=\operatorname{sqrt}\left(S^{2}\left(\frac{2 \hat{r}}{\chi_{1-\alpha, \hat{r}}^{2}}+C_{1-\alpha}\left(\frac{n+1}{(n-1)(2+\hat{r})}\right)-1\right)\right)
$$

where

$$
\begin{aligned}
& C_{\alpha(r)}=\sqrt{2} Z_{\alpha} \sqrt{r}+\frac{2}{3}\left(Z_{\alpha}^{2}-1\right) r+\frac{1}{9 \sqrt{2}}\left(Z_{\alpha}^{3}-7 Z_{\alpha}\right) r^{\frac{3}{2}} \\
& -\frac{1}{405}\left(6 Z_{\alpha}^{4}+14 Z_{\alpha}^{2}-433\right) r^{2}+\frac{1}{4860 \sqrt{2}}\left(9 Z_{\alpha}^{5}+256 Z_{\alpha}^{3}-433 Z_{\alpha}\right) r^{\frac{5}{2}}
\end{aligned}
$$

and other terms are defined as above.

\subsection{Bootstrap Cls}

Let $\mathrm{X}^{(*)}=X_{1}^{(*)}, X_{2}^{(*)}, \ldots, X_{n}^{(*)}$, where the $i^{\text {th }}$ sample is denoted by $X^{(i)}$ for $i=1,2, \ldots, B$ and $B$ is the number of bootstrap samples. Compute $\sigma$ for all bootstrap samples.

\subsubsection{Proposed Non-Parametric Bootstrap Cl}

Order the SDs of all bootstrap samples as follows: $S_{(1)}^{*} \leq S_{(2)}^{*} \leq S_{(3)}^{*} \cdots \leq S_{(B)}^{*}$.

$\mathrm{Cl}$ for population $\sigma$ :

$$
L C L=S_{[(\alpha / 2) B]}^{*} \text { and } U C L=S_{[(1-(\alpha / 2)) B]}^{*}
$$

\subsubsection{Proposed Parametric Bootstrap $\chi^{2} \mathrm{Cl}$ \\ $\mathrm{Cl}$ for population $\sigma$ :}

$\mathrm{LCL}=S \sqrt{(n-1) / \chi_{\alpha / 2,(n-1)}^{* 2}}$ and

$\mathrm{UCL}=S \sqrt{(n-1) / \chi_{1-(\alpha / 2),(n-1)}^{* 2}}$

where $\chi_{\alpha / 2}^{* 2}$ and $\chi_{1-(\alpha / 2)}^{* 2}$ are $(\alpha / 2)^{t h}$ and $(1-(\alpha / 2))^{\text {th }}$ sample quintiles of $\chi^{2}=\frac{(n-1) S^{2}}{\hat{\sigma}_{B}}$, $\hat{\sigma}_{B}=\sqrt{\frac{1}{B-1} \sum_{i=1}^{B}\left(\bar{x}_{i}^{*}-\overline{\bar{x}}\right)^{2}}$ is the bootstrap SD, $\bar{x}_{i}^{*}$ is the $i^{\text {th }}$ bootstrap sample mean and $\overline{\bar{x}}$ is the bootstrap mean.

\subsubsection{Cojbasic and Tomovic (CT) Cl}

Based on t-statistic, [13] proposed the following nonparametric bootstrap t $\mathrm{Cl}$ for $\sigma$ :

$$
I_{\text {boot }}=S^{2}-\hat{t}^{(\alpha)} \sqrt{\operatorname{var}\left(S^{2}\right)}
$$

where $S^{2}$ is the sample variance, $\hat{t}^{(\alpha)}$ is the $\alpha$ percentile of $\mathrm{T}^{*}$ defined as $T^{*}=\frac{S^{2^{*}}-S^{2}}{\sqrt{\operatorname{var}\left(S^{2^{*}}\right)}}, \mathrm{S}^{2^{*}}$ is a bootstrap replication of statistic $S^{2}$ and $\operatorname{var}\left(S^{2}\right)$ is a consistent estimator of $S^{2}$, defined by $2 \sigma^{4} /(n-1)$. 


\section{SIMULATION STUDY}

Since, a theoretical comparison among selected intervals is not possible, a simulation study has been conducted to compare performances of the selected intervals. The simulation plan and discussion of the results are given in this section.

\subsection{Simulation Plan}

MATLAB [14] programming language was used to run the simulations and to make necessary tables.

The flowchart of our simulation is as follows:

We used random samples of sizes $n=15,25,50$ and 100. Random samples are generated from various symmetric (light and heavy tailed) and skewed distributions which are:

(a) Standard normal distribution $\mathrm{N}(0,1)$ with skewness zero.

(b) $\quad \operatorname{Gamma}(3,1)$ distribution with skewness 2.

(c) t-distribution with $8 \mathrm{df}$ with skewness 2 .

(d) $\quad \operatorname{Beta}(5,1)$ distribution with skewness -1.8232 .

(e) Lognormal distribution with mean 3 and SD 0.75 with skewness 3.2629 .

(f) Chi-square distribution with $7 \mathrm{df}$ with skewness 1.0690 .

We used 5000 replications and 1500 bootstrap samples for each sample size $\mathrm{n}$. The most common $95 \% \mathrm{Cl}(\alpha=0.05)$ for confidence coefficient is used. It is well known that if data are from a symmetric distribution (or $\mathrm{n}$ is large), coverage probability will be exact or close to (1- $\alpha)$. So coverage probability is a useful criterion for evaluating $\mathrm{Cl}$. Another criterion is the width of $\mathrm{Cl}$. A shorter width (tighter $\mathrm{Cl}$ ) gives a better $\mathrm{Cl}$. It is obvious that when coverage probability is the same, a smaller width indicates that method is appropriate for the specific sample. In order to compare performance of various intervals, the following criteria are considered: (i) Coverage probabilities (below, coverage, and above), (ii) Mean width and (iii) SD of width. Below (above) rate of a $\mathrm{Cl}$ is the fraction out of 5000 samples that resulted in an interval that lies entirely above (below) the true value of $\sigma$. The coverage probability is found as sum of lower rate and upper rate and then subtracted from total probability of 1. Simulation results are reported in Tables 1-6 respectively for different selected distributions and for better understanding graphical methods are selected.

\subsection{Simulation Results and Discussions}

In Tables 1-6, we have tabulated performances of the selected intervals for standard normal, gamma, $t$, beta, lognormal and chi-square distributions. For better understanding, the cover rate is presented in Figures 16.

Table 1 and the Figure 1 present the simulation results for the normal distribution case. It is not a surprise to see the ordinary chi square interval to perform the best with very close coverage to the nominal value across all sample sizes selected. Nonparametric confidence interval seems to have the second best performance in the normally distributed data. In general, it is apparent that coverage probability increases as the sample size increases. On the other hand, the AdjDF confidence interval seems to underestimate the coverage probability for all sample sizes in the normally distributed data.

Table 2 and Figure 2 present the simulation results for the Gamma distribution case. The robust, nonparametric and the parametric bootstrap confidence intervals seem to perform better especially for large sample size $(n=100)$. The classical chi-square, the AdjDF, the B-C and the CT nonparametric bootstrap seems to underestimate the coverage probability for data from the Gamma distribution.

Table 3 and Figure 3 present the simulation results for the $t$ distribution with 8 degrees of freedom. For sample size at least 50 , Bonett and nonparametric confidence interval seems to have better performance compared to others, with the AdjDF and B-C still underestimating the coverage probability. In Table 4 and Figure 4, we have presented simulation results for the Beta $(5,1)$ distribution. In the presence of the negatively skewed data, we observe that for sample sizes of at least 50, Bonett, Steve, Ln_A, nonparametric and parametric bootstrap intervals have confidence cover rate close to the nominal level. We see that with increasing sample sizes, confidence probability close to the nominal level. The AdjDF and $B-C$ intervals cover rate found below the nominal level.

Table $\mathbf{5}$ and the Figure $\mathbf{5}$ report the simulation results for the lognormal $(3,0.75)$ distribution in the case of highly skewed data. It is easy to see that in the presence of highly skewed data, the robust, the 
Table 1: Coverage Probabilities for N(0,1) Distribution with Skewness 0

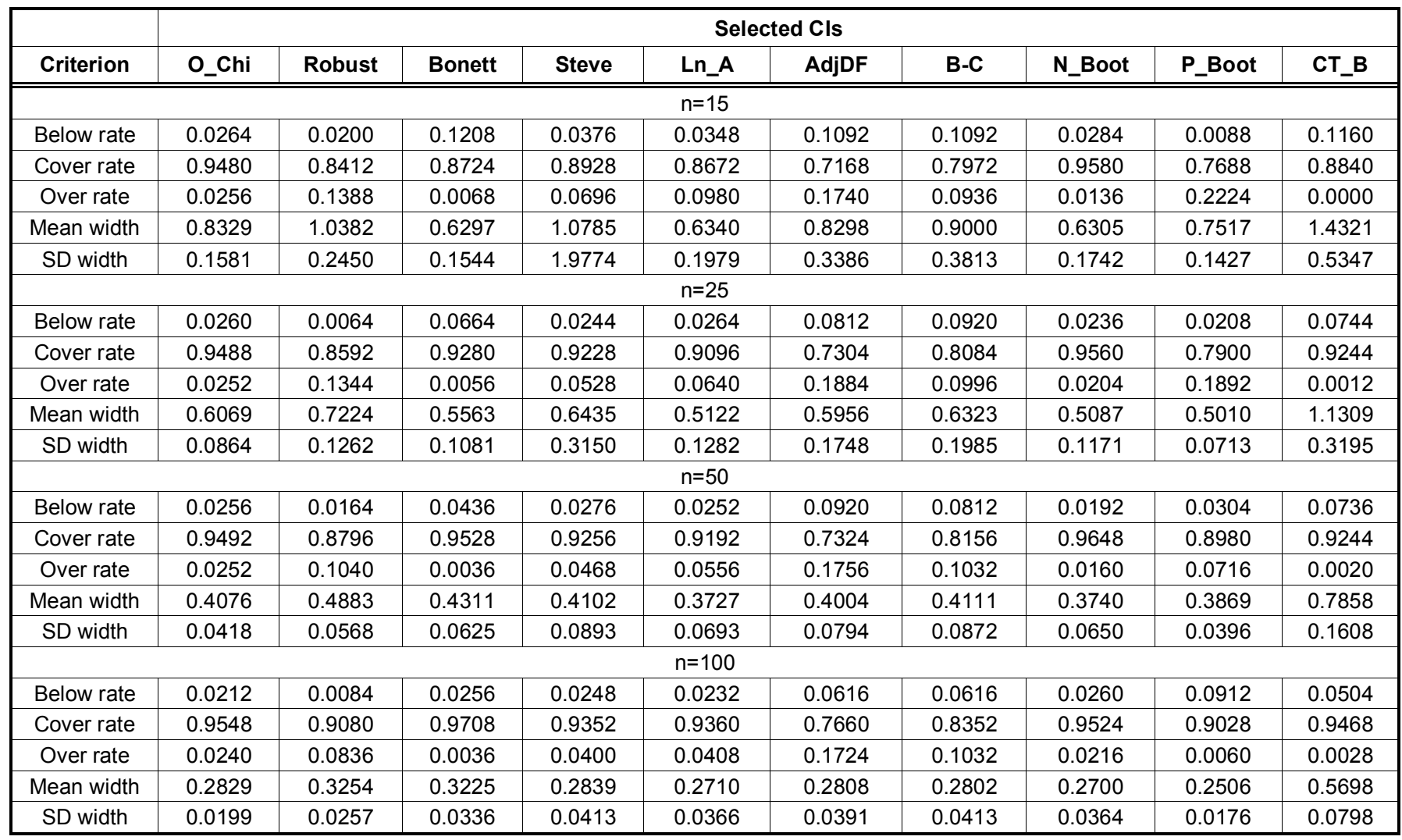

Notes: O_Chi: Ordinary Chi-Square given in (1), Ln_A: Ln Asympt given in (6), B-C: B-C AdjDF given (8), N_boot: Non-parametric Bootstrap given (9), P_Boot: Parametric Bootstrap given in (10) and CT_B: Cojbasic and Tomovic Bootstrap given (11).

Table 2: Coverage Probabilities for Gamma (3,1) Distribution with Skewness 2

\begin{tabular}{|c|c|c|c|c|c|c|c|c|c|c|}
\hline \multirow[b]{2}{*}{ Criterion } & \multicolumn{10}{|c|}{ Selected Cls } \\
\hline & O_Chi & Robust & Bonett & Steve & Ln_A & AdjDF & B-C & N_Boot & P_Boot & CT_B \\
\hline \multicolumn{11}{|c|}{$n=15$} \\
\hline Cover rate & 0.6684 & 0.5312 & 0.6848 & 0.7892 & 0.7232 & 0.6092 & 0.6420 & 0.9596 & 0.5564 & 0.3816 \\
\hline Over rate & 0.1548 & 0.0116 & 0.0332 & 0.0964 & 0.2336 & 0.3664 & 0.3336 & 0.0224 & 0.4420 & 0.4664 \\
\hline Mean width & 2.4183 & 2.0736 & 2.3142 & 5.6337 & 2.5880 & 4.4530 & 4.7586 & 2.3897 & 7.4903 & 12.1519 \\
\hline Below rate & 0.1768 & 0.3184 & 0.2028 & 0.0980 & 0.0296 & 0.0024 & 0.0024 & 0.0224 & 0.0116 & 0.1288 \\
\hline Cover rate & 0.7008 & 0.6700 & 0.7780 & 0.8208 & 0.7860 & 0.6156 & 0.6428 & 0.9376 & 0.7140 & 0.5988 \\
\hline Over rate & 0.1224 & 0.0008 & 0.0192 & 0.0812 & 0.1844 & 0.3820 & 0.3548 & 0.0400 & 0.2744 & 0.2724 \\
\hline Mean width & 1.7816 & 1.4084 & 2.2379 & 4.8849 & 2.3184 & 3.2109 & 3.4970 & 2.2018 & 3.6328 & 9.3015 \\
\hline SD width & 0.4844 & 0.3415 & 1.1357 & 8.6046 & 1.3475 & 2.6915 & 2.8628 & 1.1391 & 0.9877 & 5.3407 \\
\hline Mean width & 1.2041 & 0.9492 & 1.8702 & 3.3232 & 1.8320 & 2.1591 & 2.3344 & 1.7957 & 1.8317 & 7.0344 \\
\hline SD width & 0.2346 & 0.1596 & 0.7854 & 5.9067 & 0.8649 & 1.2837 & 1.4334 & 0.7681 & 0.3569 & 2.8281 \\
\hline \multicolumn{11}{|c|}{$n=100$} \\
\hline Below rate & 0.1496 & 0.0188 & 0.0996 & 0.0652 & 0.0172 & 0.0000 & 0.0000 & 0.0268 & 0.0196 & 0.3112 \\
\hline Cover rate & 0.7452 & 0.9812 & 0.8904 & 0.8864 & 0.8760 & 0.6860 & 0.7036 & 0.9436 & 0.9580 & 0.6608 \\
\hline Over rate & 0.1052 & 0.0000 & 0.0100 & 0.0484 & 0.1068 & 0.3140 & 0.2964 & 0.0296 & 0.0224 & 0.0280 \\
\hline Mean width & 0.8440 & 0.6297 & 1.5048 & 1.8336 & 1.4417 & 1.5695 & 1.6593 & 1.3960 & 1.3418 & 4.9846 \\
\hline SD width & 0.1199 & 0.0738 & 0.5275 & 1.3004 & 0.5606 & 0.7000 & 0.7901 & 0.4941 & 0.1907 & 1.4385 \\
\hline
\end{tabular}

Notes: See Table 1. 
Table 3: Coverage Probabilities for $\mathbf{t}$ Distribution with 8 df and Skewness 2

\begin{tabular}{|c|c|c|c|c|c|c|c|c|c|c|}
\hline \multirow[b]{2}{*}{ Criterion } & \multicolumn{10}{|c|}{ Selected CIs } \\
\hline & O_Chi & Robust & Bonett & Steve & Ln_A & AdjDF & B-C & N_Boot & P_Boot & CT_B \\
\hline \multicolumn{11}{|c|}{$n=15$} \\
\hline Cover rate & 0.8736 & 0.8724 & 0.8108 & 0.8752 & 0.8316 & 0.7440 & 0.8024 & 0.9436 & 0.5816 & 0.5892 \\
\hline Over rate & 0.0660 & 0.0896 & 0.0096 & 0.0704 & 0.1388 & 0.2520 & 0.1936 & 0.0308 & 0.3844 & 0.3552 \\
\hline Mean width & 0.9472 & 1.1172 & 0.7719 & 1.6057 & 0.8095 & 1.1377 & 1.2388 & 0.7695 & 0.7509 & 1.9770 \\
\hline Below rate & 0.0516 & 0.0488 & 0.1096 & 0.0468 & 0.0228 & 0.0096 & 0.0096 & 0.0288 & 0.0072 & 0.1112 \\
\hline Cover rate & 0.8844 & 0.8876 & 0.8820 & 0.8940 & 0.8728 & 0.7196 & 0.7800 & 0.9524 & 0.8656 & 0.8488 \\
\hline Over rate & 0.0640 & 0.0636 & 0.0084 & 0.0592 & 0.1044 & 0.2708 & 0.2104 & 0.0188 & 0.1272 & 0.0400 \\
\hline Mean width & 0.6935 & 0.7807 & 0.7091 & 1.1304 & 0.6875 & 0.8385 & 0.9046 & 0.6511 & 0.8742 & 1.4628 \\
\hline SD width & 0.1257 & 0.1509 & 0.2298 & 3.0172 & 0.2735 & 0.4204 & 0.4736 & 0.2150 & 0.1584 & 0.5432 \\
\hline Mean width & 0.4726 & 0.5315 & 0.5754 & 0.6401 & 0.5276 & 0.5842 & 0.6133 & 0.6466 & 0.3665 & 1.0958 \\
\hline SD width & 0.0640 & 0.0689 & 0.1908 & 0.4586 & 0.2121 & 0.3110 & 0.3413 & 0.2206 & 0.0497 & 0.3309 \\
\hline \multicolumn{11}{|c|}{$n=100$} \\
\hline Below rate & 0.0488 & 0.0724 & 0.0468 & 0.0376 & 0.0212 & 0.0572 & 0.0572 & 0.0232 & 0.0456 & 0.1204 \\
\hline Cover rate & 0.8976 & 0.9028 & 0.9472 & 0.9232 & 0.9136 & 0.5928 & 0.6556 & 0.9508 & 0.8992 & 0.8580 \\
\hline Over rate & 0.0536 & 0.0248 & 0.0060 & 0.0392 & 0.0652 & 0.3500 & 0.2872 & 0.0260 & 0.0552 & 0.0216 \\
\hline Mean width & 0.3260 & 0.3529 & 0.4318 & 0.4238 & 0.3855 & 0.4047 & 0.4122 & 0.3678 & 0.3505 & 0.7504 \\
\hline SD width & 0.0302 & 0.0317 & 0.1023 & 0.2323 & 0.1111 & 0.1277 & 0.1419 & 0.0936 & 0.0325 & 0.1412 \\
\hline
\end{tabular}

Notes: See Table 1.

Table 4: Coverage Probabilities for Beta(5,1) Distribution with Skewness $\mathbf{- 1 . 8 2 3 2}$

\begin{tabular}{|c|c|c|c|c|c|c|c|c|c|c|}
\hline \multirow[b]{2}{*}{ Criterion } & \multicolumn{10}{|c|}{ Selected Cls } \\
\hline & O_Chi & Robust & Bonett & Steve & Ln_A & AdjDF & B-C & N_Boot & P_Boot & CT_B \\
\hline \multicolumn{11}{|c|}{$\mathrm{n}=15$} \\
\hline Cover rate & 0.8716 & 0.5892 & 0.7864 & 0.8336 & 0.7920 & 0.6488 & 0.7236 & 0.9432 & 0.6196 & 0.7824 \\
\hline Over rate & 0.0544 & 0.0000 & 0.0136 & 0.0832 & 0.1668 & 0.2452 & 0.1704 & 0.0284 & 0.3604 & 0.0000 \\
\hline Mean width & 0.1156 & 0.1171 & 0.0956 & 0.2150 & 0.1006 & 0.1453 & 0.0505 & 0.0996 & 0.1364 & 0.0281 \\
\hline Below rate & 0.0696 & 0.2652 & 0.1200 & 0.0544 & 0.0328 & 0.0908 & 0.0908 & 0.0220 & 0.0392 & 0.1968 \\
\hline Cover rate & 0.8724 & 0.7312 & 0.8672 & 0.8724 & 0.8532 & 0.6716 & 0.7356 & 0.9504 & 0.8932 & 0.8032 \\
\hline Over rate & 0.0580 & 0.0036 & 0.0128 & 0.0732 & 0.1140 & 0.2376 & 0.1736 & 0.0276 & 0.0676 & 0.0000 \\
\hline Mean width & 0.0848 & 0.0817 & 0.0881 & 0.1462 & 0.0857 & 0.1054 & 0.1139 & 0.0841 & 0.1425 & 0.0202 \\
\hline SD width & 0.0155 & 0.0176 & 0.0263 & 0.2009 & 0.0319 & 0.0486 & 0.0550 & 0.0281 & 0.0260 & 0.0073 \\
\hline Mean width & 0.0574 & 0.0554 & 0.0705 & 0.0779 & 0.0649 & 0.0715 & 0.0752 & 0.0640 & 0.0623 & 0.0151 \\
\hline SD width & 0.0072 & 0.0080 & 0.0156 & 0.0355 & 0.0176 & 0.0215 & 0.0243 & 0.0165 & 0.0078 & 0.0038 \\
\hline \multicolumn{11}{|c|}{$n=100$} \\
\hline Below rate & 0.0604 & 0.1160 & 0.0372 & 0.0272 & 0.0212 & 0.0280 & 0.0280 & 0.0172 & 0.0072 & 0.1152 \\
\hline Cover rate & 0.8832 & 0.8548 & 0.9560 & 0.9312 & 0.9268 & 0.7108 & 0.7636 & 0.9656 & 0.9760 & 0.8708 \\
\hline Over rate & 0.0564 & 0.0292 & 0.0529 & 0.0416 & 0.0520 & 0.2612 & 0.2084 & 0.0172 & 0.0168 & 0.0140 \\
\hline Mean width & 0.0398 & 0.0367 & 0.0083 & 0.0511 & 0.0473 & 0.0496 & 0.0453 & 0.0470 & 0.0592 & 0.0110 \\
\hline SD width & 0.0035 & 0.0037 & 0.0038 & 0.0112 & 0.0091 & 0.0100 & 0.0123 & 0.0088 & 0.0053 & 0.0020 \\
\hline
\end{tabular}

Notes: See Table 1. 
Table 5: Coverage Probabilities for Lognormal (3,0.75) Distribution with Skewness 3.2629

\begin{tabular}{|c|c|c|c|c|c|c|c|c|c|c|}
\hline \multirow[b]{2}{*}{ Criterion } & \multicolumn{10}{|c|}{ Selected Cls } \\
\hline & O_Chi & Robust & Bonett & Steve & Ln_A & AdjDF & B-C & N_Boot & P_Boot & CT_B \\
\hline \multicolumn{11}{|c|}{$\mathrm{n}=15$} \\
\hline Cover rate & 0.4912 & 0.5736 & 0.5952 & 0.7452 & 0.6444 & 0.5772 & 0.6080 & 0.9608 & 0.5132 & 0.4640 \\
\hline Over rate & 0.1916 & 0.0048 & 0.0312 & 0.0920 & 0.3188 & 0.4228 & 0.3920 & 0.0164 & 0.4712 & 0.1164 \\
\hline Mean width & 17.7645 & 14.5656 & 18.2198 & 40.2524 & 20.8625 & 38.9665 & 41.1572 & 19.3970 & 90.2271 & 704.1570 \\
\hline Below rate & 0.3024 & 0.3176 & 0.2964 & 0.1528 & 0.0324 & 0.0000 & 0.0000 & 0.0204 & 0.0000 & 0.3776 \\
\hline Cover rate & 0.5364 & 0.6820 & 0.6728 & 0.7660 & 0.6952 & 0.5792 & 0.6328 & 0.9480 & 0.5956 & 0.5108 \\
\hline Over rate & 0.1612 & 0.0004 & 0.0308 & 0.0812 & 0.2724 & 0.4208 & 0.3672 & 0.0316 & 0.4044 & 0.1116 \\
\hline Mean width & 13.2422 & 9.9536 & 18.7886 & 40.5456 & 20.0204 & 31.4447 & 33.7228 & 18.3004 & 34.6845 & 596.1258 \\
\hline SD width & 5.0325 & 2.2631 & 13.5612 & 62.0221 & 16.0029 & 41.2178 & 41.0870 & 12.6592 & 13.1812 & 587.3846 \\
\hline Mean width & 9.1533 & 6.6811 & 17.3491 & 34.3152 & 17.5468 & 23.0647 & 25.0409 & 16.1378 & 14.3138 & 433.2265 \\
\hline SD width & 2.6139 & 1.0451 & 11.4679 & 59.6452 & 12.5365 & 24.3287 & 25.5348 & 9.8277 & 4.0876 & 284.9240 \\
\hline \multicolumn{11}{|c|}{$n=100$} \\
\hline Below rate & 0.2444 & 0.0008 & 0.1716 & 0.1040 & 0.0156 & 0.0000 & 0.0000 & 0.0296 & 0.0176 & 0.4296 \\
\hline Cover rate & 0.6468 & 0.9592 & 0.8164 & 0.8404 & 0.8072 & 0.6756 & 0.6416 & 0.9468 & 0.9368 & 0.5220 \\
\hline Over rate & 0.1088 & 0.0400 & 0.0120 & 0.0556 & 0.1772 & 0.3244 & 0.3584 & 0.0236 & 0.0456 & 0.0484 \\
\hline Mean width & 6.4167 & 4.4301 & 14.6205 & 26.8553 & 14.4405 & 16.9179 & 18.2981 & 13.4852 & 17.7357 & 287.7666 \\
\hline SD width & 1.3627 & 0.4745 & 8.3297 & 70.4268 & 8.7546 & 13.3320 & 14.7615 & 7.1230 & 3.7666 & 135.2810 \\
\hline
\end{tabular}

Notes: See Table 1

Table 6: Coverage Probabilities for Chi-Square Distribution with $7 \mathrm{df}$ and Skewness 1.0690

\begin{tabular}{|c|c|c|c|c|c|c|c|c|c|c|}
\hline \multirow[b]{2}{*}{ Criterion } & \multicolumn{10}{|c|}{ Selected Cls } \\
\hline & O_Chi & Robust & Bonett & Steve & Ln_A & AdjDF & B-C & N_Boot & P_Boot & CT_B \\
\hline \multicolumn{11}{|c|}{$\mathrm{n}=15$} \\
\hline Below rate & 0.0724 & 0.1528 & 0.1840 & 0.0648 & 0.0360 & 0.0764 & 0.0764 & 0.0264 & 0.1532 & 0.1488 \\
\hline Cover rate & 0.8508 & 0.8444 & 0.8024 & 0.8528 & 0.8172 & 0.5656 & 0.6044 & 0.9572 & 0.8204 & 0.8328 \\
\hline Over rate & 0.0768 & 0.0028 & 0.0136 & 0.0824 & 0.1468 & 0.3580 & 0.3192 & 0.0164 & 0.0264 & 0.0184 \\
\hline Mean width & 3.0788 & 3.4975 & 2.5061 & 5.5108 & 2.6211 & 3.7393 & 4.0583 & 2.5767 & 2.4435 & 21.2189 \\
\hline SD width & 0.7257 & 0.8745 & 0.9375 & 15.5594 & 1.2302 & 2.7713 & 2.9290 & 1.0379 & 0.5760 & 10.1998 \\
\hline \multicolumn{11}{|c|}{$n=25$} \\
\hline Below rate & 0.0700 & 0.0700 & 0.1316 & 0.0620 & 0.0276 & 0.0392 & 0.0392 & 0.0228 & 0.0836 & 0.1488 \\
\hline Cover rate & 0.8592 & 0.8592 & 0.8564 & 0.8688 & 0.8424 & 0.6300 & 0.6564 & 0.9540 & 0.8592 & 0.8364 \\
\hline Over rate & 0.0708 & 0.0708 & 0.0120 & 0.0692 & 0.1300 & 0.3308 & 0.3044 & 0.0232 & 0.0572 & 0.0148 \\
\hline Mean width & 2.2420 & 2.4122 & 2.3007 & 4.1157 & 2.2285 & 2.7416 & 2.9575 & 2.1686 & 5.0641 & 15.8237 \\
\hline SD width & 0.4268 & 0.4348 & 0.8170 & 9.2627 & 0.9808 & 1.5614 & 1.7454 & 0.8474 & 0.9639 & 6.1527 \\
\hline \multicolumn{11}{|c|}{$\mathrm{n}=50$} \\
\hline Below rate & 0.0596 & 0.0444 & 0.0868 & 0.0552 & 0.0240 & 0.0100 & 0.0100 & 0.0180 & 0.0716 & 0.1156 \\
\hline Cover rate & 0.8768 & 0.8928 & 0.9052 & 0.8976 & 0.8720 & 0.6700 & 0.7000 & 0.9540 & 0.8592 & 0.8464 \\
\hline Over rate & 0.0636 & 0.0628 & 0.0080 & 0.0472 & 0.1040 & 0.3200 & 0.2900 & 0.0280 & 0.0692 & 0.0380 \\
\hline Mean width & 1.5237 & 1.5237 & 1.8825 & 2.1810 & 1.7332 & 1.9215 & 2.0238 & 1.6930 & 1.5027 & 11.1350 \\
\hline SD width & 0.2070 & 0.2070 & 0.5673 & 1.7014 & 0.6389 & 0.8118 & 0.9183 & 0.5671 & 0.2041 & 3.0723 \\
\hline \multicolumn{11}{|c|}{$n=100$} \\
\hline Below rate & 0.0512 & 0.0608 & 0.0580 & 0.0496 & 0.0156 & 0.0060 & 0.0060 & 0.0220 & 0.0000 & 0.1456 \\
\hline Cover rate & 0.8880 & 0.9144 & 0.9348 & 0.9116 & 0.9084 & 0.7136 & 0.7660 & 0.9592 & 0.8812 & 0.8528 \\
\hline Over rate & 0.0608 & 0.0248 & 0.0072 & 0.0388 & 0.0760 & 0.2804 & 0.2280 & 0.0188 & 0.1188 & 0.0016 \\
\hline Mean width & 1.0569 & 1.0912 & 1.4382 & 1.4200 & 1.2940 & 1.3614 & 1.3921 & 1.2733 & 1.0750 & 7.7087 \\
\hline SD width & 0.1029 & 0.0928 & 0.3456 & 0.5167 & 0.3783 & 0.4243 & 0.4686 & 0.3516 & 0.1047 & 1.5074 \\
\hline
\end{tabular}

Notes: See Table 1. 
$n=15-n=25-n=50 \quad n=100$

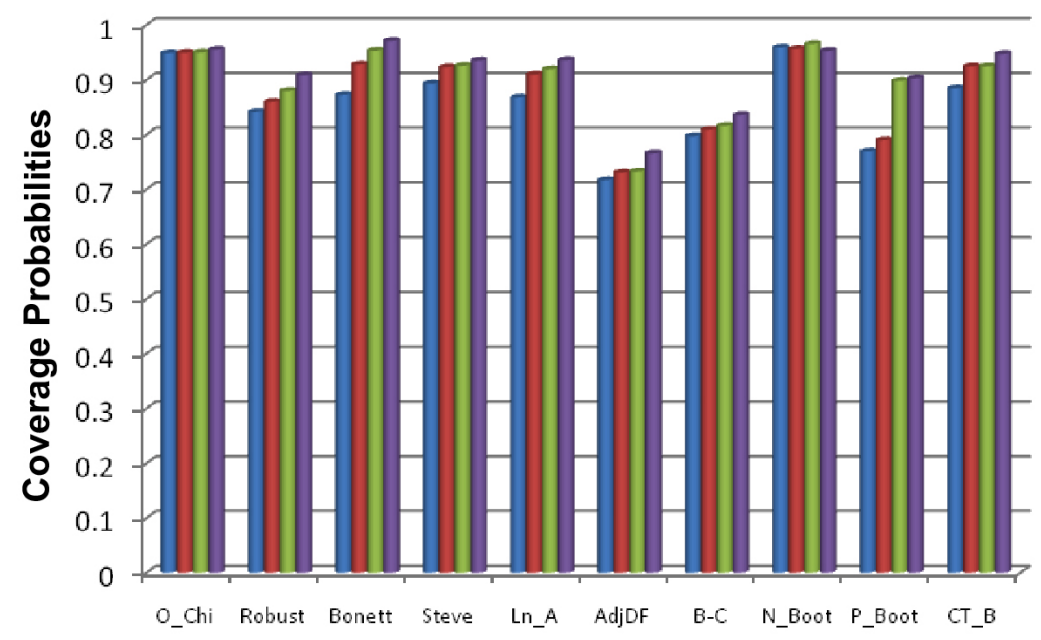

Figure 1: Coverage Probabilities for selected intervals for data from the $N(0,1)$ distribution.

$n=15 \square n=25-n=50 \quad n=100$

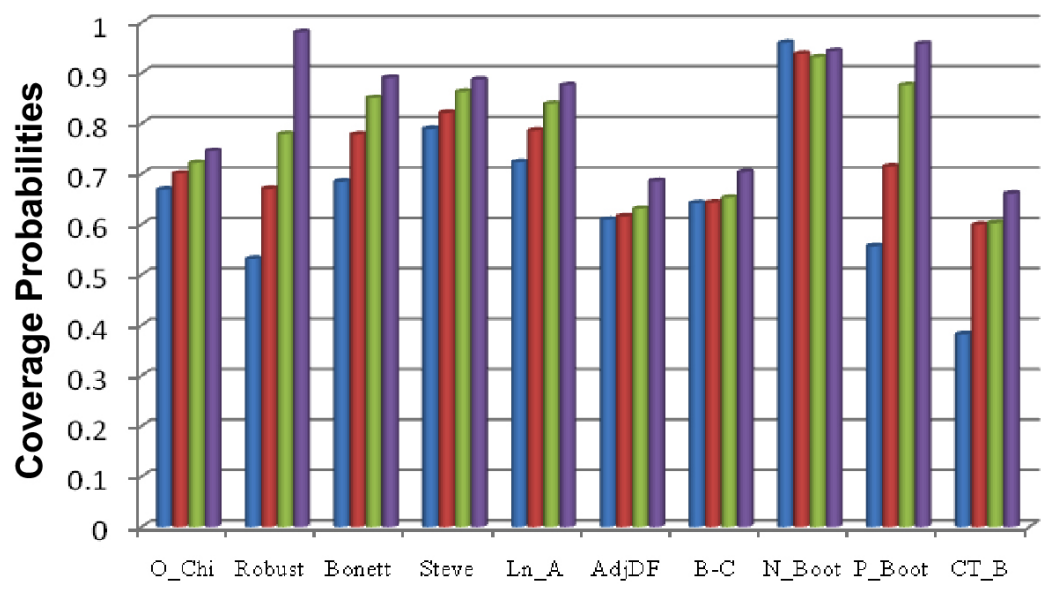

Figure 2: Coverage Probabilities for selected intervals for data from the $G(3,1)$ distribution.

$n=15-n=25 \quad n=50 \quad n=100$

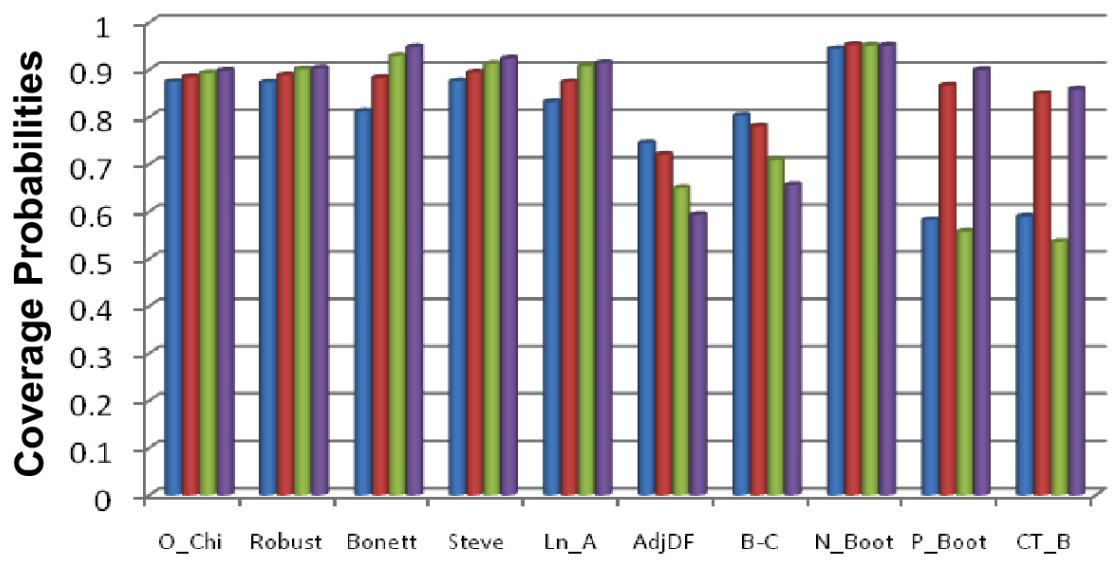

Figure 3: Coverage Probabilities for selected intervals for data from the t distribution with $8 \mathrm{df}$. 
$n=15-n=25-n=50 \quad n=100$

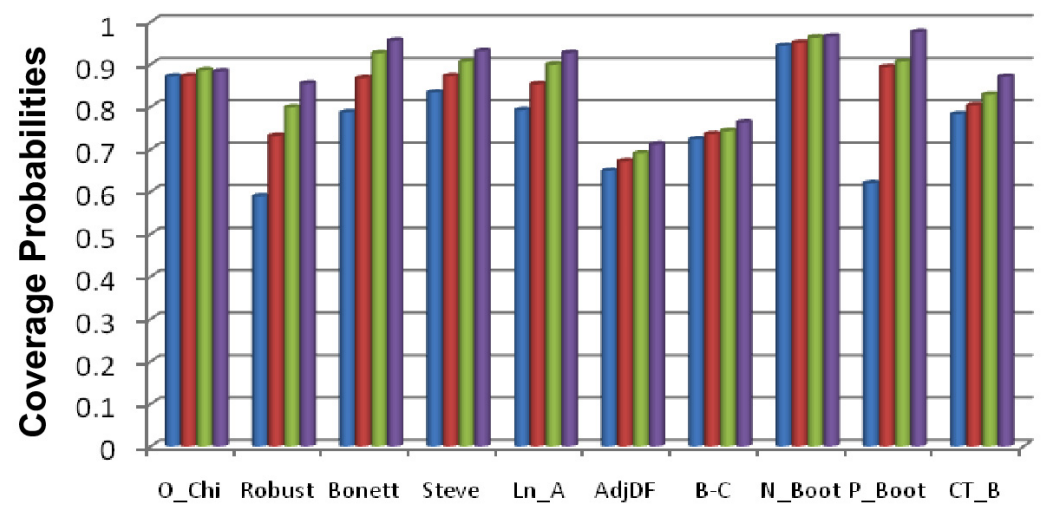

Figure 4: Coverage Probabilities for selected intervals for data from the Beta( 5,1$)$ distribution.

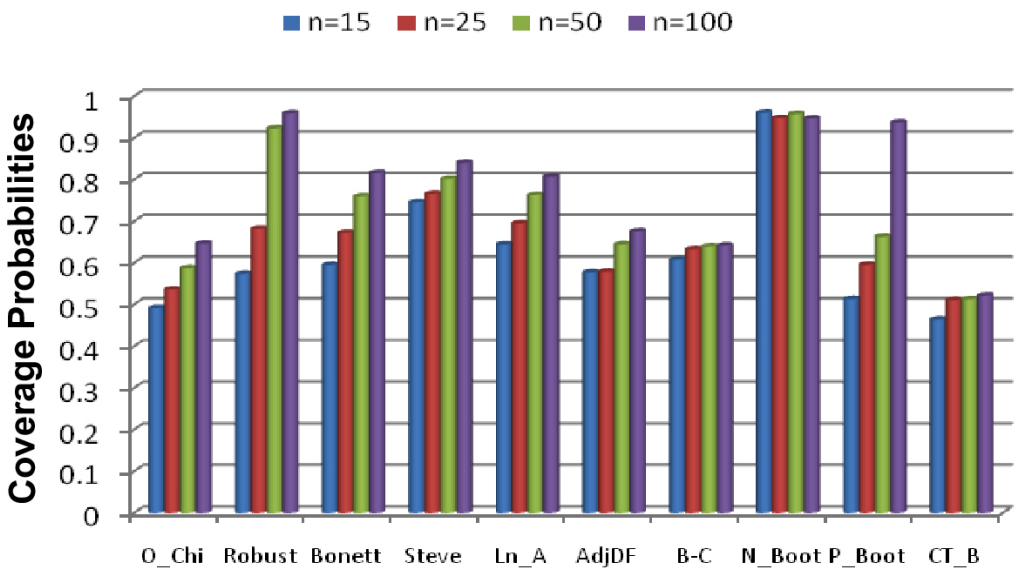

Figure 5: Coverage Probabilities for selected intervals for data from the lognormal $(3,0.75)$ distribution.

non-parametric and the parametric intervals have coverage rate close to 0.95 for large samples. The classical chi-square, AdfDF, B-C and CT nonparametric bootstrap intervals underestimates the coverage probability.

Table 6 and Figure 6 report simulation results for the chi-square distribution with $7 \mathrm{df}$. It is observed from these results that with increasing sample sizes, coverage probability for selected intervals converge to the nominal probability of 0.95 except the AdjDF and the $\mathrm{B}-\mathrm{C}$ intervals.

These two intervals although have an increase in coverage probability with an increase in the sample size $n$, still underestimate coverage rate.

Overall, according to our simulation results, Cls such as robust interval, Bonnet interval, Steve interval, Ln_Asymptinterval and the nonparametric bootstrap interval have the best performance and thus can be recommended for researchers.

\section{REAL DATA APPLICATION}

To illustrate the findings of the paper, two health related data are analyzed in this section.

\subsection{Example 1}

A study by Aizenberg et al. [15] examined the efficacy of sildenafil, a potent phospho-diesterase inhibitor in the treatment of elderly men with erectile dysfunction induced by antidepressant treatment for major depressive disorder. The ages of 10 enrollees were:

$$
74,81,70,70,74,77,76,70,71,72
$$

Suppose we are interested to construct most common $95 \% \mathrm{Cl}$ for $\sigma$ of ages of elderly men in the population. Sample mean, sample standard deviation and sample skewness of age are 73.5000, 3.6591 and 0.7961 respectively. The statistical software EasyFit developed by mathwave data analysis and simulations company was used to fit 60 distributions to this data 


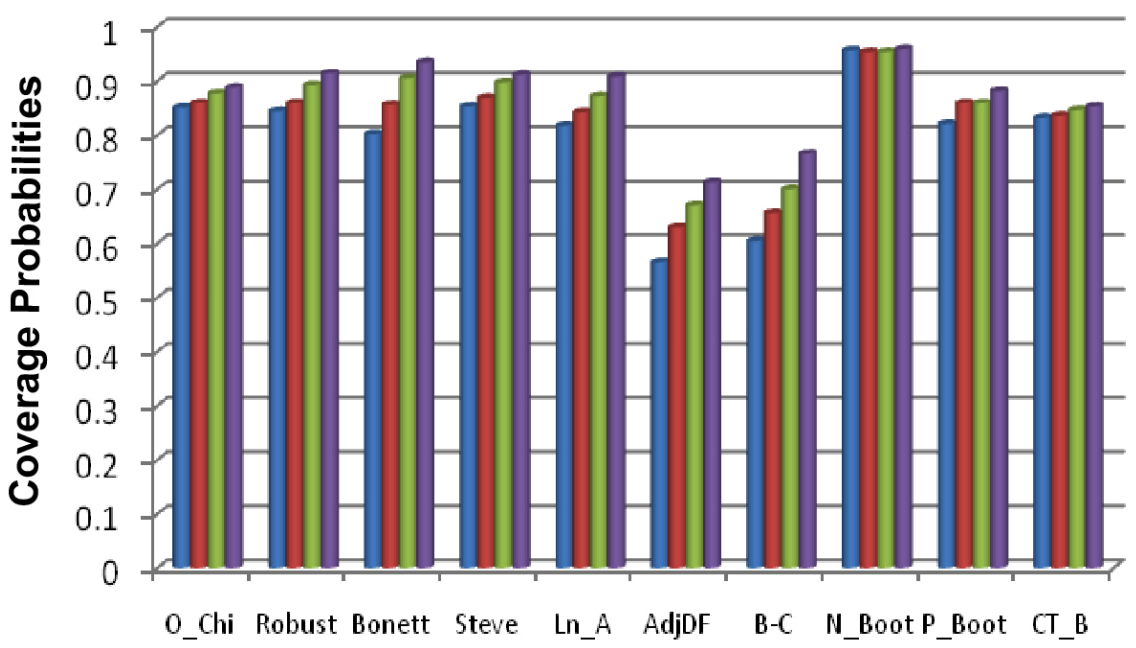

Figure 6: Coverage Probabilities for selected intervals for data from the chi-square distribution with $7 \mathrm{df}$.

set. A goodness-of-fit procedure is performed on all 60 distributions and their performances are ranked based on the goodness-of-fit measure. According to mathwave, the distribution which best fits the age data is the Gumbel Max distribution which ranked number one using the Kolmogrov-Simrnov test. The location and scale parameters of the Gumbel Max distribution obtained are $\mu=71.853$ and $\sigma=2.853$, respectively. The variance of the Gumbel Max distribution is given by $\frac{\pi^{2}}{6} \sigma^{2}$. By substituting the scale estimate $\sigma$, an estimate of the Gumbel Max population standard deviation is $\sqrt{13.389}=3.659$. The resulting $95 \% \quad \mathrm{Cls}$ and corresponding confidence widths are provided in Table $\mathbf{7}$ and in the Figure $\mathbf{7}$ for better understanding. A careful inspection of those intervals reveals that all of them captured the true parameter value of 3.659 except the robust interval. Among the intervals that captured the true parameter value, Bonett interval has the narrowest width followed by the nonparametric interval and the Ln_A interval.

Table 7:Confidence Intervals Estimate of $\sigma$ for Age Data Example

\begin{tabular}{|c|c|c|}
\hline Method & $\mathbf{9 5 \%} \mathbf{~ C l}$ & Width \\
\hline \hline O_Chi & $(2.5168,6.6801)$ & 4.1632 \\
\hline Robust & $(4.4911,12.9234)$ & 8.4323 \\
\hline Bonett & $(2.3065,4.8535)$ & 2.5470 \\
\hline Steve & $(2.7303,8.1034)$ & 5.3731 \\
\hline Ln_A & $(2.4575,5.4481)$ & 2.9905 \\
\hline AdjDF & $(2.4716,7.0100)$ & 4.5384 \\
\hline B-C & $(2.4716,7.4909)$ & 5.0194 \\
\hline N_Boot & $(1.9120,4.7842)$ & 2.8723 \\
\hline P_Boot & $(2.8374,7.2850)$ & 4.4476 \\
\hline CT_B & $(1.1279,5.0016)$ & 3.8737 \\
\hline
\end{tabular}

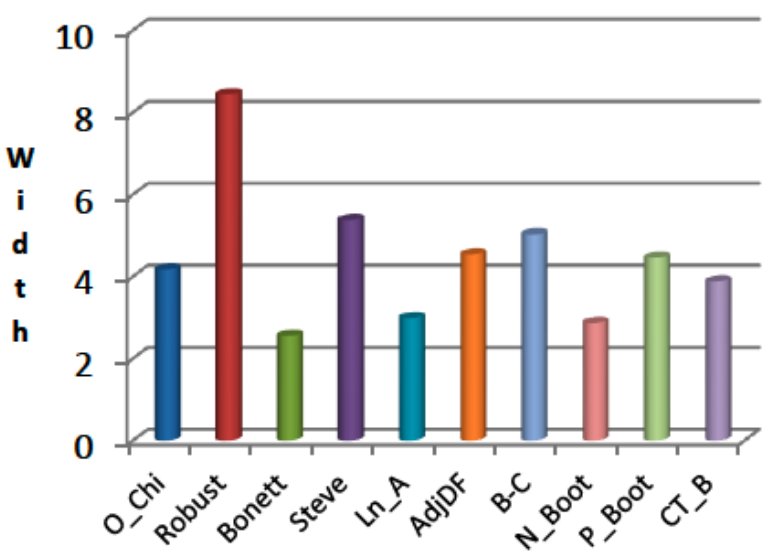

Figure 7: Confidence interval width for age data example.

\subsection{Example 2}

A study tested the tumorigenesis of a drug. Rats were randomly selected from litters and given the drug. The times of tumor appearance (TTA) were recorded as follows [16]:

$101,104,104,77,89,88,104,96,82,70,89,91,39$, $103,93,85,104,104,81,67,104,104,104,87,104$, $89,78,104,86,76,103,102,80,45,94,104,104,76$, $80,72,73$

Sample mean, sample standard deviation and skewness of the TTA data are 88.7805, 15.9930 and 1.21718 respectively. Similar to example 1, using EasyFit software, the distribution with the best fit was found to be the general Pareto distribution with parameters: location, scale, and shape given by $\mu=51.381, \sigma=125.8$, and $\xi=-2.3636$, respectively. The variance of the general Pareto distribution is given by 
Variance $=\frac{\sigma^{2}}{(1-\xi)^{2}(1-2 \xi)}, \xi<\frac{1}{2}$

Thus, the variance of the general Pareto distribution is 244.2363, and hence an estimated population standard deviation of the general Pareto distribution is 15.628. We are interested inconstructing $95 \% \mathrm{Cl}$ for $\sigma$ of the time until a tumor appearance. The resulting $\mathrm{Cl}$ and corresponding confidence widths are reported in Table 8 and widths of $\mathrm{Cl}$ are presented in Figure 8 for better understanding. A careful inspection of the intervals in Table 8 reveals that all confidence intervals captured the true parameter value of 15.628 , except the robust interval. Among the nineintervals captured the true parameter value, we found that CT_B has the shortest width, followed by robust and the chisquare intervals, respectively.

Table 8: Confidence Intervals Estimates of $\sigma$ for the TA Data

\begin{tabular}{|c|c|c|}
\hline Method & $95 \%$ Cl & Width \\
\hline \hline O_Chi & $(13.1304,20.4631)$ & 7.3326 \\
\hline Robust & $(16.1379,23.2821)$ & 7.1442 \\
\hline Bonett & $(11.5967,21.0496)$ & 9.4529 \\
\hline Steve & $(12.7921,24.1947)$ & 11.4026 \\
\hline Ln_A & $(12.0687,21.1932)$ & 9.1245 \\
\hline AdjDF & $(12.3689,22.6357)$ & 10.2668 \\
\hline B-C & $(12.3689,23.3562)$ & 10.9873 \\
\hline N_Boot & $(11.2756,20.2355)$ & 8.9599 \\
\hline P_Boot & $(12.6710,22.4034)$ & 9.7324 \\
\hline CT_B & $(12.6797,19.4429)$ & 6.7632 \\
\hline
\end{tabular}

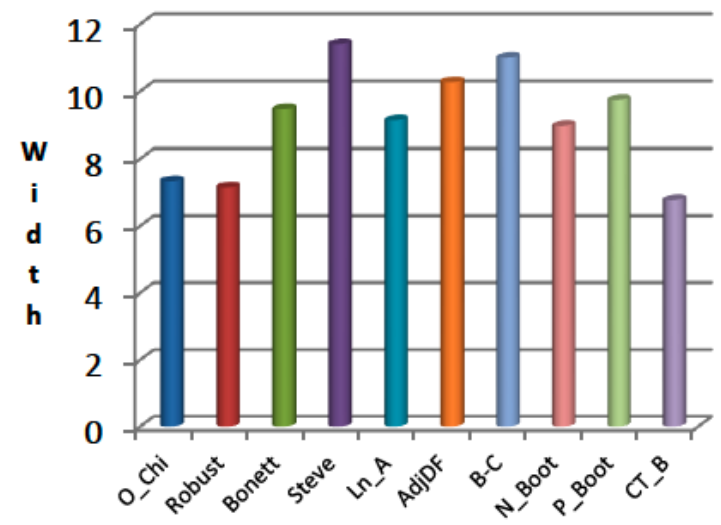

Figure 8: $95 \%$ interval width for the TA data.

\section{CONCLUSION}

This article generalizes the work conducted by [2]. An extensive simulation study has been conducted to compare the performance of several interval estimators for the population standard deviation. We considered the classical chi-square interval, the robust interval, the Bonett interval, the Steve interval, and three intervals proposed by [1] and three versions of bootstrap intervals. Various symmetric (light and heavy tailed) data and skewed data (positive and negatively distributed data) are evaluated. Our simulation results advise that the robust interval, the Bonnet interval, the Steve interval, the Ln_A interval, the nonparametric bootstrap intervals can be recommended for use by researchers. Some Real-life data are considered to illustrate the application of the proposed confidence intervals which also supported the simulation study to some extent.

We hope that findings of this paper will be helpful for applied researchers who want to select the proper method(s) for confidence interval for the parameter, population standard deviation.

\section{ACKNOWLEDGEMENTS}

The authors are thankful to the editor and referee for their valuable comments that improved the presentation of the paper greatly.

\section{REFERENCES}

[1] Hummel R, Banga S, Hettmansperger TP. Better confidence intervals for the variance in a random sample. Minitab Technical Report 2005; Retrieved from: http://www.minitab.com/support/documentation/answers/One Variance.pdf

[2] Abu-Shawiesh M, Banik S, Kibria BMG. A simulation study on some Confidence intervals for estimating the population standard deviation. SORT 2011; 35(2): 83-102.

[3] Tukey JW. A survey of sampling from contaminated distributions. In Olkin I, et al. Eds. Contributions to Probability and Statistics, Essays in Honor of Harold Hotelling. Stanford: Stanford University Press 1960; pp. 448-485.

[4] Banik S, Kibria BMG. Comparison of some parametric and nonparametric type one sample confidence intervals for estimating the mean of a positively skewed distribution. Commun Stat Simul Comput 2010a; 39: 361-389. http://dx.doi.org/10.1080/03610910903474530

[5] Banik S, Kibria BMG. Comparison of some test statistics for testing the mean of a right skewed distribution. J Stat Theory Appl 2010b; 8: 77-90.

[6] Shi W, Kibria BMG. On some confidence intervals for estimating the mean of a skewed population. Int J Math Educ Sci Technol 2007; 38: 412-421. http://dx.doi.org/10.1080/00207390601116086

[7] Baklizi A, Kibria BMG. One and two sample confidence intervals for estimating the mean of skewed populations: An empirical comparative study. J Appl Stat 2009; 36: 601-609. http://dx.doi.org/10.1080/02664760802474298

[8] Gross AM. Confidence interval robustness with long-tailed symmetric distributions. J Am Stat Assoc 1976; 71: 409-416. http://dx.doi.org/10.1080/01621459.1976.10480359 
[9] Bonett DG. Approximate confidence interval for standard deviation of non-normal distributions. Comput Stat Data Anal 2006; 50: 775-782.

http://dx.doi.org/10.1016/i.csda.2004.10.003

[10] Lehman EL. Testing Statistical Hypothesis, John Wiley, New York 1986.

http://dx.doi.org/10.1007/978-1-4757-1923-9

[11] Rousseuw PJ, Croux C. Alternatives to the median absolute deviation. J Am Stat Assoc 1993; 88: 1273-1283. http://dx.doi.org/10.1080/01621459.1993.10476408

[12] Steve A. Mathematical Statistics. Prentice Hall College Division 1990.

[13] Cojbasic V, Tomovic A. Nonparametric confidence intervals for population variance of one sample and the difference of variances of two samples. Comput Stat Data Anal 2007; 51: 5562-5578.

http://dx.doi.org/10.1016/i.csda.2007.03.023

[14] MATLAB. version 7.10.0. Natick, Massachusetts: The MathWorks Inc 2010.

[15] Aizenberg D. Weizman A, Barak Y. Sildenafil for selective serotonin reuptake inhibitor induced erectile dysfunction in elderly male depressed patients. J Sex Marital Ther 2003; 29: 297-303. http://dx.doi.org/10.1080/00926230390195533

[16] Mantel N, Bohidar NR, Ciminera JL. Analysis of littermatched time-to-response data, with modifications for recovery of interlitter information. J Cancer Res 1977; 37: 3863-3868. 